\title{
Article \\ Using GIS to Estimate Population at Risk Because of Residence Proximity to Asbestos Processing Facilities in Colombia
}

\author{
Benjamin Lysaniuk $^{1}\left(\mathbb{D}\right.$, María Fernanda Cely-García ${ }^{2}\left(\mathbb{D}\right.$, Margarita Giraldo $^{2}$, Joan M. Larrahondo ${ }^{3}$ (D), \\ Laura Marcela Serrano-Calderón ${ }^{3}$, Juan Carlos Guerrero-Bernal ${ }^{4}$, Leonardo Briceno-Ayala ${ }^{5}$ (D), \\ Esteban Cruz Rodriguez ${ }^{4}$ and Juan Pablo Ramos-Bonilla ${ }^{2, *}$
}

check for

updates

Citation: Lysaniuk, B.; Cely-García, M.F.; Giraldo, M.; Larrahondo, J.M.; Serrano-Calderón, L.M.;

Guerrero-Bernal, J.C.; Briceno-Ayala,

L.; Cruz Rodriguez, E.;

Ramos-Bonilla, J.P. Using GIS to

Estimate Population at Risk Because

of Residence Proximity to Asbestos

Processing Facilities in Colombia. Int

J. Environ. Res. Public Health 2021, 18,

13297. https://doi.org/10.3390/

ijerph182413297

Academic Editors: Lucia Fazzo,

Carolina Mensi and Paul

B. Tchounwou

Received: 16 October 2021

Accepted: 5 November 2021

Published: 17 December 2021

Publisher's Note: MDPI stays neutral with regard to jurisdictional claims in published maps and institutional affiliations.

Copyright: (C) 2021 by the authors Licensee MDPI, Basel, Switzerland. This article is an open access article distributed under the terms and conditions of the Creative Commons Attribution (CC BY) license (https:// creativecommons.org/licenses/by/ $4.0 /$ )
1 IRD (MàD by CNRS)_UMR Prodig, 93222 Aubervilliers, France; Benjamin.Lysaniuk@cnrs.fr

2 Departamento de Ingeniería Civil y Ambiental, Universidad de Los Andes, Bogotá 111711, Colombia; mf.cely46@uniandes.edu.co (M.F.C.-G.); mm.giraldo337@uniandes.edu.co (M.G.)

3 Departamento de Ingeniería Civil, Facultad de Ingeniería, Pontificia Universidad Javeriana, Bogotá 110231, Colombia; jlarrahondo@javeriana.edu.co (J.M.L.); lauramserranoc@gmail.com (L.M.S.-C.)

4 Facultad de Estudios Internacionales, Políticos y Urbanos (FEIPU), Universidad del Rosario, Bogotá 111711, Colombia; juan.guerrero@urosario.edu.co (J.C.G.-B.); esteban.cruz@urosario.edu.co (E.C.R.)

5 Escuela de Medicina y Ciencias de la Salud, Universidad del Rosario, Bogotá 111711, Colombia; leonardo.briceno@urosario.edu.co

* Correspondence: jramos@uniandes.edu.co

Abstract: The recent enactment of the law banning asbestos in Colombia raises a significant number of challenges. The largest factories that have historically processed asbestos include five asbestoscement facilities located in the cities of Sibaté (Cundinamarca), Cali (Valle del Cauca), and Barranquilla (Atlántico), and Manizales (Caldas), which has two, as well as a friction products facility in Bogotá D.C. An asbestos chrysotile mine has also operated in Colombia since 1980 in Campamento (Antioquia). In the framework of developing the National Asbestos Profile for Colombia, in this study, we estimated the population residing in the vicinity of asbestos processing plants or the mine and, therefore, potentially at risk of disease. Using a geographic information system, demographic data obtained from the last two general population censuses were processed to determine the number of people living within the concentric circles surrounding the asbestos facilities and the mine. In previous studies conducted in different countries of the world, an increased risk of asbestos-related diseases has been reported for people living at different distance bands from asbestos processing facilities. Based on these studies, circles of 500, 1000, 2000, 5000, and 10,000 m radii, centered on the asbestos processing facilities and the mine that operated in Colombia, were combined with the census data to estimate the number of people living within these radii. Large numbers of people were identified. It is estimated that in 2005, at the country level, 10,489 people lived within $500 \mathrm{~m}$ of an asbestos processing facility or mine. In 2018, and within a distance of 10,000 $\mathrm{m}$, the number of people was $6,724,677$. This information can aid public health surveillance strategies.

Keywords: asbestos; environmental exposure; population; geographic information system; Colombia

\section{Introduction}

Asbestos minerals are classified into two groups, namely amphiboles and serpentines [1]. Amphiboles include five asbestos minerals (amosite, actinolite, anthophyllite, tremolite, and crocidolite), and serpentines include chrysotile, which is currently the type of asbestos most extensively used worldwide [1-4]. Because of their physical and chemical characteristics, asbestos minerals have been used in many products and in several industrial sectors [5,6]. All types of asbestos are carcinogenic to humans (i.e., type I carcinogens), and exposure to this material causes diseases such as mesothelioma, lung cancer, laryngeal cancer, ovarian cancer, and asbestosis [7,8]. However, it is difficult to calculate the burden of disease related to the population attributable fraction of these multifactorial diseases 
because of limitations in the asbestos-related environmental and health data [9], especially in low- and middle-income countries. Previous studies have shown that the risk for lung cancer from asbestos exposure is variable according to the type of exposure (environmental, domestic, or occupational) [10], level of exposure, the relative toxicity of asbestos type [11], awareness of the health and socio-economic impact of asbestos use and bans [12], and the level of release of fibers from materials, among other variables [13]. Unfortunately, this type of information is not always available in low- and middle-income countries, as is the case for Colombia. The highest levels of asbestos exposure and the burden of asbestosrelated diseases occur primarily in occupationally exposed workers, but it is important to acknowledge that the role of non-occupational exposure cannot be neglected [1,14].

Despite the negative effects of asbestos on human health, products containing asbestos are still sold in many countries. As of 2021, only 67 countries, a group that Colombia joined recently, have banned this mineral [15]. In July 2019, Law 1968 was enacted in Colombia, banning the use of all asbestos types [16]. The law establishes that starting 1 January 2021, all activities related to asbestos are prohibited, including its mining, production, selling, importation, distribution, and exportation. This is undoubtedly one of the most important achievements in recent years in the field of public health in Colombia, as it helps to curb the growth of the asbestos problem. However, it is important to understand that Law 1968/19 by itself is not the solution to the asbestos problem, and among other issues, the asbestos products that have been distributed throughout the country for 78 years still represent a major health threat and an important technical and economic challenge. In fact, Law 1968/19 stipulates that the Colombian government has five years from the enactment of the law to formulate a strategy to address this problem. One of the conditions making it more difficult for strategy designs to face the challenges imposed by the use of asbestos in Colombia is the lack of information regarding the magnitude and characteristics of the problem.

The World Health Organization (WHO) and the International Labour Organization (ILO) have recommended that countries implement National Programs for the Elimination of Asbestos-Related Diseases (NPEAD), a strategy designed to address the asbestos problem at the country level [17]. The preparation of NPEAD begins with the development of the National Asbestos Profile (NAP), which includes 18 sections that describe the country's legislative context, the production and trade of mineral asbestos and asbestos-containing products, the industries with the highest risk of exposure and high-risk workers, the incidence and prevalence of asbestos-related diseases, the occupational exposure limits of the country, the studies conducted on the topic in the country, and estimated economic losses due to asbestos-related diseases [17]. This study is part of an effort to develop the NAP of Colombia, which is currently in development by a multidisciplinary group of engineers, geologists, physicians, geographers, and political scientists from three Colombian Universities and a French public research institution.

Abundant scientific literature shows that populations that are settled nearby industrial asbestos processing facilities and mines are at excessive risk of developing asbestosrelated diseases. The cases of Casale Monferrato (Italy) [18,19], Amagasaki (Japan) [20,21], Wittenoom (Australia) [22], Libby (USA) [23]—among other examples—are particularly well documented.

Six major facilities and an asbestos mine, shown in Table 1, have operated in Colombia and were the focus of the current study.

The asbestos processing facilities include five asbestos cement facilities that manufactured construction products (e.g., corrugated asbestos cement sheets, pipes for water supply and sanitation systems, and water storage tanks), and one friction products plant that manufactured products for the automotive sector (e.g., brake pads, brake linings, brake blocks, and clutch discs). There is also a chrysotile asbestos mine located in Campamento (Department of Antioquia).

Using a geographic information system (GIS) to process census records for Colombia from different years, in this study, we estimated the number of people from the general 
population living in distance bands from asbestos processing facilities and the asbestos mine at which an elevated risk of asbestos-related diseases (ARD) has been reported in the scientific literature.

Table 1. The six facilities and the mine included in this study.

\begin{tabular}{ccccc}
\hline Name & $\begin{array}{c}\text { Municipality } \\
\text { (and Department) }\end{array}$ & $\begin{array}{c}\text { Type of } \\
\text { Facility }\end{array}$ & Production & Starting Date \\
\hline Eternit Colombiana SA & Sibaté (Cundinamarca) & Plant & Asbestos cement & 1942 \\
Eternit Atlántico SA & Barranquilla (Atlántico) & Plant & Asbestos cement & 1944 \\
Eternit Pacífico SA & Yumbo (Valle del Cauca) & Plant & Asbestos cement & 1944 \\
Incolbest SA & Bogotá (Bogotá DC) & Plant & Friction products & 1960 \\
Etex Colombia SA & Manizales (Caldas) & Plant & Asbestos cement & 1967 \\
Toptec SA & Manizales (Caldas) & Plant & Asbestos cement & 1982 \\
Minera Las Brisas & Campamento (Antioquia) & Mine & Chrysotile & 1980 \\
\hline
\end{tabular}

\section{Materials and Methods}

To determine the distances around asbestos facilities or asbestos mines at which an elevated risk of asbestos-related diseases has been observed, a literature review was performed to identify studies that had conducted this type of analysis. The literature review was conducted in PubMed at the end of 2020, using the search words "asbestos", "distance", "environmental exposure" and "plant OR factory", with no limits on the publication year.

Using a GIS, the distances identified in the literature review were cross-checked with demographic information obtained from the last two general censuses of the Colombian population (i.e., 2005 and 2018) developed by the National Administrative Department of Statistics (Departamento Administrativo Nacional de Estadística-DANE) [24]. These censuses are the only ones available online. To estimate the population living within these distances, the National Geostatistical Framework of Colombia (Marco Geoestadistico Nacional-MGN) [25] was used. This database is organized following the political and administrative divisions of Colombia and includes demographic information that is very specific from a geographical perspective.

All administrative subdivisions of Colombia are characterized by a unique identifier appearing both in the MGN and in the DANE databases. Thus, it was possible to integrate the relevant census data into each polygon representing the administrative units of interest for 2005 and 2018. Demographic data in Colombia is divided between urban and rural areas. Considering the radii used, it was, therefore, necessary to integrate the urban and rural areas to minimize the risk of population loss in the estimates. To do this, the smallest administrative level for each of the censuses was used (i.e., sections and/or sectors) according to the following classification:

- $\quad$ For the year 2005: population counts by five-year age groups for urban sections and rural sectors.

- $\quad$ For the year 2018: population counts by five-year age groups for urban and rural sections.

The urban sections for the 2005 census correspond to the second smallest level of data in urban areas. The city blocks (i.e., manzanas) are the most precise areas, but because of the large amount of data that the DANE servers had to process at the city block level, an error message was always generated when conducting the analysis at this level. Furthermore, since large amounts of data were missing from rural sections for 2005, the next size level for rural areas (i.e., rural sectors) was used. The urban and rural sections of the 2018 census correspond to the most precise level of data in both types of areas for that year. The procedure for obtaining the data used in the analysis is detailed in the Supplementary Material, Figure S1.

As explained before, the analysis was conducted in the regions where asbestos facilities or the mine are located, including the departments of Antioquia (Las Brisas Mine in the municipality of Campamento), Atlántico (Eternit in the municipality of Barranquilla), Bogotá D.C. (Incolbest in the Capital District), Caldas (Etex and Toptec in the municipality 
of Manizales), Cundinamarca (Eternit in the municipality of Sibaté), and Valle del Cauca (Eternit in the municipality of Yumbo). Data from the Departments of Magdalena and Tolima were also integrated into the analysis of the populations around Eternit, Barranquilla and Etex, Manizales respectively, since small areas of these departments were located within some radii of interest.

After compiling all the data, it was processed with MGN shapefiles using Arcgis 10.8 (CEsri). The procedure used included the following steps (the complete procedure is detailed in the Supplementary Material, Figure S2):

- Location of the asbestos facilities and the mine using satellite images integrated into a GIS via a web server and the creation of a point for each site.

- Creation of buffer zones using the distances identified in the literature review.

- Calculation of the individual area of the MGN polygons (area A).

- Use of the "intersection" tool under Arcgis between each buffer zone and the polygons of the urban sections (2005 and 2018), rural sectors (2005), and rural sections (2018).

- Calculation of the area of the polygons resulting from the intersection between the buffers and the MGN layers (area B).

- Calculation of the ratio between area B and area A. This ratio was applied to the census populations in cases in which polygons of the MGN were not fully covered by a buffer.

- Integration of the census data by table joining the DANE data and the "buffer/MGN polygons" intersection layers.

- Exportation of the attribute tables in text format, followed by post-processing (i.e., the application of the ratio between area $\mathrm{B}$ to area $\mathrm{A}$ and the corresponding population), and estimation of the number of people residing there, categorized in five-year age groups, within the concentric radii surrounding the asbestos processing facilities and the mine. This procedure was done for both years 2005 and 2018. When fractional numbers were obtained for the population estimates, the value was rounded to the nearest integer, which may result in some slight differences in the calculations of row and column sums in the tables presented in the results.

The Integrated Information System of Social Protection (SISPRO), the official database of morbidity and mortality managed by the Colombian Ministry of Health and Social Protection, was also consulted to determine if it was possible to find cases of mesothelioma, a sentinel disease of asbestos exposure, in the six municipalities where the analysis was conducted. SISPRO was accessed on 5 October 2021. Three Excel pivot tables were built, filtering by the "main diagnosis" of pleural, peritoneal, and pericardial mesothelioma (i.e., CIE10 450, 451, and 452, respectively). Searches were made at the municipality level for Campamento (Antioquia), Barranquilla (Atlántico), Bogotá (DC), Manizales (Caldas), Sibaté (Cundinamarca), and Yumbo (Valle del Cauca). Gender, date of healthcare provided, and age at the moment of the healthcare provided (i.e., by 5-year age groups) were also included in the search. The numerical variable used was "number of people served", which shows the number of persons served by the healthcare system at a particular moment and locations with specific diseases within a specific window of time, clarifying that because of how the database works, more than one healthcare visit may be reported for the same person. Since the information in SISPRO can only be consulted at the municipality level, and the exact current and past locations of residence of the cases are not available in the database, it was not possible to conduct a more detailed geographic analysis of the exact location of the mesothelioma cases identified by SISPRO.

\section{Results}

In the literature review, seven studies that analyzed the risk of developing malignant mesothelioma at different distances from an industrial emission source point, generally an asbestos-cement factory, were identified $[19,21,26-30]$. The studies are presented in Table 2. The distances considered in these articles refer to radii relative to the circles centered on the point of emission (e.g., an asbestos-cement facility). For mining activities, several 
articles were identified for Libby (USA) [31,32], Wittenoom (Australia) [33,34], and South Africa $[35,36]$. Unfortunately, an analysis of the risk of asbestos-related diseases in relation to the distance to the mine was not conducted, and it was not possible to identify this type of analysis in regions where asbestos mines have operated. In the case of Libby, Noonan [37] found that local residents suffered from elevated mesothelioma incidence rates between 1.5 and $11 \mathrm{~km}$ from the mine. However, the study recognized that it was not possible to determine if the elevated rates were the result of atmospheric dispersion from the mine or from asbestos residues deposited in residential areas, as it was previously discussed for the same site [32]. Thus, for the current study, to estimate the number of people that live in the vicinity of the asbestos mine, the same distance bands around asbestos processing facilities for which the literature indicates an increased risk of malignant mesothelioma were applied.

In summary, for the current analysis, the distances used were radii of 500, 1000, 2000, 5000 , and 10,000 $\mathrm{m}$, as shown in Table 2, which also includes the health risk estimates reported in the studies. In Table 2, it is possible to observe great variations in the risk estimates. When comparing the magnitude of such risk estimates, there are no clear tendencies among the studies. This is expected since the study designs, local contexts, and asbestos facility characteristics differ, conferring unique features for each study and, consequently, unique findings.

Cross-referencing the demographic data from the DANE with that of the MGN was done to estimate the populations living within distance bands around the six asbestos processing facilities and the mine, and the results are presented in Table 3 (with 2005 census data) and Table 4 (with 2018 census data). Figure 1 presents two maps of the buffer zones created, and the associated mesothelioma risk reported for each distance band, in the municipality of Yumbo, surrounding an asbestos cement plant (Eternit Pacífico), and in Manizales, surrounding two asbestos cement plants (Etex and Toptec).

For both 2005 and 2018, the population potentially at risk was-like the Colombian population-relatively young. In 2005, $27.5 \%$ of the people living within a radius of 10,000 $\mathrm{m}$ around the sites were between 0 and 14 years old, which is similar-for the same yearto the percentage of this age group for the entire Colombian population (28.94\%) [38]. In 2018 , this proportion of very young people living within a $10,000 \mathrm{~m}$ radius fell to $20 \%$, which is slightly lower compared to the percentage of people in this age group for the entire Colombian population (23.07\%) [38].

It is also important to highlight that six of the seven locations analyzed were either within an urban center or in close proximity to urban centers, with the exception of the mine, which is located in a remote rural area. This explains the large number of people identified, which obviously increased as the radii increased. With the exception of the mine in Campamento, with a total of 2724 inhabitants in 2005 and 8472 inhabitants in 2018 living within a $10 \mathrm{~km}$ radius of the mine (Tables 3 and 4), all the other sites analyzed resulted in population numbers in the order of hundreds of thousands or even millions of people. For the 2005 census, the number of people living in a $10 \mathrm{~km}$ radius of an asbestos processing facility ranged from 282,479 around Etex (Manizales) to 3,346,920 around Incolbest (Bogotá), and for the 2018 census, the range was from 312,615 around Etex (Manizales) to 3709,324 around Incolbest (Bogotá) (Tables 3 and 4).

Another important aspect of this analysis is that there were two regions that had two asbestos processing plants; one region was the city of Manizales (Caldas), with the asbestos cement plants of Etex and Toptec, and the other was the Department of Cundinamarca where the Capital District of Bogotá is located, with the Incolbest friction products plant in Bogotá and the asbestos cement plant of Eternit in Sibaté. Thus, the number of people living within the area of influence of two plants was identified. In conducting this analysis, it was determined that 282,175 inhabitants lived less than 10,000 $\mathrm{m}$ from both the Etex and Toptec plants in Manizales in 2005 (312,554 people in 2018), and 38,728 people lived less than 10,000 $\mathrm{m}$ from both the Incolbest (Bogotá) and Eternit (Sibaté) plants in 2005 (51,831 people in 2018) (Tables 5 and 6). 
Table 2. Summary of distances used in the current study.

\begin{tabular}{|c|c|c|c|c|c|c|c|c|c|c|c|}
\hline \multirow[t]{2}{*}{ Article Reference } & \multirow{2}{*}{$\begin{array}{l}\text { Asbestos- } \\
\text { Related } \\
\text { Diseases } \\
\text { Studied } \\
\end{array}$} & \multirow{2}{*}{$\begin{array}{c}\text { Sources of } \\
\text { Environmental } \\
\text { Exposure }\end{array}$} & \multirow[t]{2}{*}{ Asbestos Type } & \multirow{2}{*}{$\begin{array}{l}\text { Risk-Related } \\
\text { Indicator }\end{array}$} & \multicolumn{6}{|c|}{ Relationship between Risk and Distance to the Plant } & \multirow[t]{2}{*}{ Observations } \\
\hline & & & & & $<500 \mathrm{~m}$ & $500 \mathrm{~m}$ & $1000 \mathrm{~m}$ & $2000 \mathrm{~m}$ & $5000 \mathrm{~m}$ & $10,000 \mathrm{~m}$ & \\
\hline Vimercati et al., 2020 & $\begin{array}{l}\text { Epithelioid } \\
\text { mesothelioma }\end{array}$ & $\begin{array}{l}\text { Asbestos } \\
\text { cement plant }\end{array}$ & $\begin{array}{l}\text { Amosite and } \\
\text { crocidolite: } 20 \% \text {; } \\
\text { chrysotile: } 80 \%\end{array}$ & $\begin{array}{l}\text { (1) Adjusted risks } \\
\text { of EM (OR) by } \\
\text { distance and } \\
\text { exposure length (2) } \\
\text { Adjusted risks of } \\
\text { EM (OR) by } \\
\text { distance and } \\
\text { latency length }\end{array}$ & & $\begin{array}{c}(<550 \mathrm{~m})(1) \\
\mathrm{OR}=1.47 \\
(0.262-8.248) / /(2) \\
\mathrm{OR}=1.771 \\
(0.82-9.805) . \\
\text { Compared to people } \\
\text { living at }>1000 \mathrm{~m}\end{array}$ & $\begin{array}{c}(550-1000 \mathrm{~m})(1) \\
\mathrm{OR}=1.87 \\
(0.35-9.905) / /(2) \\
\mathrm{OR}=2.038 \\
(0.371-11.19) . \\
\text { Compared to people } \\
\text { living at }>1000 \mathrm{~m}\end{array}$ & & & & $\begin{array}{l}\text { Apulia Mesothelioma } \\
\text { Registry. There is an } \\
\text { exposure reconstruction. } \\
\text { They looked at the records of } \\
2236 \text { cases between } 1998 \text { and } \\
\text { 2019. The } 71 \text { cases analyzed } \\
\text { were only environmentally } \\
\text { exposed. The distance of } \\
550-1000 \text { m is a finding of the } \\
\text { study, based on the living } \\
\text { locations of the } 71 \text { cases. } 3000 \\
m \text { was the largest distance } \\
\text { that cases lived from the } \\
\text { factory, but there was no risk } \\
\text { estimate for } 3000 \mathrm{~m} \text {. }\end{array}$ \\
\hline Tarrés et al., 2012 & $\begin{array}{c}\text { Malignant } \\
\text { pleural } \\
\text { mesothelioma }\end{array}$ & $\begin{array}{l}\text { Asbestos } \\
\text { cement plant }\end{array}$ & $\begin{array}{l}\text { Amosite: } 5 \% ; \\
\text { crocidolite: } 15 \% \text {; } \\
\text { chrysotile: } 80 \%\end{array}$ & $\begin{array}{l}\text { Incidence rate } \\
\text { ratio }\end{array}$ & $\begin{array}{c}(<500 \mathrm{~m}) \\
\operatorname{IRR}=12.92 \\
(10.77-15.33)\end{array}$ & & & $\begin{array}{l}(>500-2000 \mathrm{~m}) \\
\operatorname{IRR}=0.70 \\
(0.28-1.44)\end{array}$ & & $\begin{array}{l}(>2000-10,000 \mathrm{~m}) \\
\operatorname{IRR}=0.23 \\
(0.02-0.72)\end{array}$ & $\begin{array}{l}\text { Clinical and epidemiological } \\
\text { data were recorded for the } 24 \\
\text { pleural mesothelioma cases. } \\
\text { Places of residence were } \\
\text { obtained from primary } \\
\text { healthcare documentation. } \\
\text { Concentric circles were used } \\
\text { for the analysis. }\end{array}$ \\
\hline $\begin{array}{l}\text { Kurumatani, } \\
\text { Kumagai, } 2008\end{array}$ & $\begin{array}{l}\text { Pleural and } \\
\text { peritoneal } \\
\text { mesotheliomas } \\
\text { + pleural } \\
\text { cancers (on } \\
\text { death } \\
\text { certificate) }\end{array}$ & $\begin{array}{l}\text { Asbestos } \\
\text { cement plant }\end{array}$ & $\begin{array}{c}\text { Crocidolite and } \\
\text { chrysotile }\end{array}$ & SMR & $\begin{array}{l}\text { SMR Men }(<300 \mathrm{~m}) \\
=13.9(5.6-28.7) / / \\
\text { SMR Women } \\
(<300 \mathrm{~m})=41.4 \\
(15.2-90.1)\end{array}$ & $\begin{array}{c}\text { SMR Men }(300 \text { to } \\
600 \mathrm{~m})=5.6 \\
(2.9-9.8) / / \text { SMR } \\
\text { Women }(300 \mathrm{~m} \text { to } \\
600 \mathrm{~m})=11.3 \\
(4.5-23.3)\end{array}$ & $\begin{array}{c}\text { SMR Men }(600 \text { to } \\
900 \mathrm{~m})=2.1 \\
(0.8-4.3) / / \text { SMR } \\
\text { Women }(600 \mathrm{~m} \text { to } \\
900 \mathrm{~m})=7.2 \\
(2.9-14.8)\end{array}$ & & & & $\begin{array}{l}90 \text { cases. Distances were } \\
\text { analyzed with } 300 \mathrm{~m} \\
\text { increments. }\end{array}$ \\
\hline Musti et al., 2009 & $\begin{array}{c}\text { Malignant } \\
\text { mesothelioma }\end{array}$ & $\begin{array}{c}\text { Asbestos } \\
\text { cement plant }\end{array}$ & $\begin{array}{l}\text { Amosite: } 5 \% \text { \% } \\
\text { crocidolite: } 15 \% \text {; } \\
\text { chrysotile: } 80 \%\end{array}$ & $\begin{array}{l}\text { OR adjusted by } \\
\text { sex and age }\end{array}$ & $\begin{array}{c}(0-500 \mathrm{~m}) \\
\text { OR }=5.29 \\
(1.18-23.74) . \\
\text { Compared to a } \\
\text { distance of }>2000 \mathrm{~m}\end{array}$ & & $\begin{array}{c}(500-1000 \mathrm{~m}) \\
\mathrm{OR}=1.49 \\
(0.58-3.82) / / \\
(1000-1500 \mathrm{~m}) \\
\mathrm{OR}=1.27 \\
(0.49-3.71) . \\
\text { Compared to a } \\
\text { distance of }>2000 \mathrm{~m}\end{array}$ & $\begin{array}{l}(1500-2000 \mathrm{~m}) \\
\mathrm{OR}=2.31 \\
(0.88-6.06) \\
\text { Compared to a } \\
\text { distance of } \\
>2000 \mathrm{~m}\end{array}$ & & & $\begin{array}{l}\text { Case-control study. Regional } \\
\text { Mesothelioma Register-Bari. } \\
48 \text { cases of malignant } \\
\text { mesothelioma, } \\
\text { non-occupationally exposed, } \\
\text { and } 273 \text { controls. }\end{array}$ \\
\hline
\end{tabular}


Table 2. Cont.

\begin{tabular}{|c|c|c|c|c|c|c|c|c|}
\hline Article Reference & $\begin{array}{l}\text { Asbestos- } \\
\text { Related } \\
\text { Diseases } \\
\text { Studied }\end{array}$ & $\begin{array}{l}\text { Sources of } \\
\text { Environmental } \\
\text { Exposure }\end{array}$ & Asbestos Type & $\begin{array}{l}\text { Risk-Related } \\
\text { Indicator }\end{array}$ & Relationship between Risk and Distance to the $P$ & & & Observations \\
\hline Maule et al., 2007 & $\begin{array}{c}\text { Malignant } \\
\text { pleural } \\
\text { mesothelioma }\end{array}$ & $\begin{array}{c}\text { Asbestos } \\
\text { cement plant }\end{array}$ & $\begin{array}{l}\text { Crocidolite: } \\
10 \% \text {; “airborne } \\
\text { emissions from } \\
\text { the AC plant } \\
\text { included both } \\
\text { chrysotile and } \\
\text { crocidolite } \\
\text { fibers" }\end{array}$ & $\begin{array}{l}\text { OR adjusted by } \\
\text { sex, age, domestic } \\
\text { exposure, and } \\
\text { relatives that work } \\
\text { in the AC plant }\end{array}$ & & $\begin{array}{c}(0-3000 \mathrm{~m}) \\
\mathrm{OR}=6.8 \\
(2.4-19.5) / / \\
(3000-5000 \mathrm{~m}) \\
\mathrm{OR}=12.5 \\
(3.7-42.2)\end{array}$ & $\begin{array}{c}(5000-7000 \mathrm{~m}) \\
\mathrm{OR}=3.9(1.2-12.6) \\
/ /(7000-9000 \mathrm{~m}) \\
\text { OR }=4.2(1.1-16.5)\end{array}$ & $\begin{array}{l}\text { Case-control study. } 103 \text { cases, } \\
272 \text { controls. Casale } \\
\text { Monferrato. Piedmont } \\
\text { Mesothelioma Registry. }\end{array}$ \\
\hline Airoldi et al., 2021 & $\begin{array}{l}\text { Malignant } \\
\text { pleural } \\
\text { mesothelioma }\end{array}$ & $\begin{array}{c}\text { Asbestos } \\
\text { cement plant }\end{array}$ & $\begin{array}{c}\text { Crocidolite: } \\
10 \%\end{array}$ & $\begin{array}{l}\text { OR adjusted by } \\
\text { age, sex, type of } \\
\text { interview, and } \\
\text { domestic exposure }\end{array}$ & & $\begin{array}{c}(0-5000 \mathrm{~m}) \\
\text { OR }=12.66 \\
(4.19-38.28) . \\
\text { Compared to a } \\
\text { distance of } \\
>15,000 \mathrm{~m} \\
\end{array}$ & $\begin{array}{c}(5000-10,000 \mathrm{~m}) \\
\mathrm{OR}=7.15 \\
(1.87-27.36) . \\
\text { Compared to } \\
\text { distance of }>15,000 \\
m \\
\end{array}$ & $\begin{array}{l}\text { Case-control study. } 200 \text { cases, } \\
348 \text { controls. Casale } \\
\text { Monferrato. }\end{array}$ \\
\hline Magnani et al., 2000 & $\begin{array}{l}\text { Malignant } \\
\text { pleural } \\
\text { mesothelioma }\end{array}$ & $\begin{array}{l}\text { Asbestos } \\
\text { cement plants; } \\
\text { asbestos textiles, } \\
\text { shipyards, or } \\
\text { brakes factories }\end{array}$ & Unspecified & $\begin{array}{l}\text { OR adjusted by } \\
\text { center, sex, } \\
\text { and age }\end{array}$ & $\begin{array}{l}\text { "A high risk } \\
\text { was observed } \\
\text { for high } \\
\text { probability of } \\
\text { environmental } \\
\text { exposure (11.5 } \\
\text { (3.47-38.2))- } \\
\text { that is, subjects } \\
\text { who had lived } \\
\text { for some time } \\
\text { within } 2000 \mathrm{~m} \\
\text { of a mine or } \\
\text { asbestos } \\
\text { facility" }\end{array}$ & & & $\begin{array}{l}\text { Histologically confirmed } \\
\text { cases. Exposure classification } \\
\text { of cases. High risk of } \\
\text { exposure (not disease). }\end{array}$ \\
\hline
\end{tabular}

Table 3. Number of people living around the seven main asbestos processing/mining sites in Colombia—2005.

\begin{tabular}{|c|c|c|c|c|c|c|c|c|c|c|c|c|c|c|c|c|c|c|c|c|c|c|c|c|c|c|c|c|c|c|c|c|c|c|}
\hline \multirow[t]{2}{*}{$\begin{array}{c}\text { Age } \\
\text { Groups } \\
\text { (Yaars) } \\
\end{array}$} & \multicolumn{6}{|c|}{ Incolbest-Bogotá } & \multicolumn{4}{|c|}{ Eternit-Sibaté } & \multicolumn{6}{|c|}{ Eternit-Yumbo } & \multicolumn{4}{|c|}{ Eternit-Barranquilla } & \multicolumn{5}{|c|}{ Las Brisas-Campamento } & \multicolumn{5}{|c|}{ Toptec-Manizales } & \multicolumn{4}{|c|}{ Colombit (Etex)-Manizales } \\
\hline & $500 \mathrm{~m}$ & $1000 \mathrm{~m}$ & $2000 \mathrm{~m}$ & $5000 \mathrm{~m}$ & $10,000 \mathrm{~m}$ & $500 \mathrm{~m}$ & $1000 \mathrm{~m}$ & $2000 \mathrm{~m}$ & $5000 \mathrm{~m}$ & $10,000 \mathrm{~m}$ & $500 \mathrm{~m}$ & $1000 \mathrm{~m}$ & $2000 \mathrm{~m}$ & $5000 \mathrm{~m}$ & $10,000 \mathrm{~m}$ & $\mathrm{n} 500 \mathrm{~m}$ & $1000 \mathrm{~m}$ & $2000 \mathrm{~m}$ & $5000 \mathrm{~m}$ & $10,000 \mathrm{~m} 500 \mathrm{~m}$ & $1000 \mathrm{~m}$ & $2000 \mathrm{~m}$ & $5000 \mathrm{~m}$ & $10,000 \mathrm{~m}$ & $500 \mathrm{~m}$ & $1000 \mathrm{~m}$ & $2000 \mathrm{~m}$ & $5000 \mathrm{~m}$ & $10,000 \mathrm{~m}$ & $500 \mathrm{~m}$ & $1000 \mathrm{~m}$ & $2000 \mathrm{~m}$ & $5000 \mathrm{~m}$ & $10,000 \mathrm{~m}$ \\
\hline $\begin{array}{l}0-4 \\
5-9 \\
10-14 \\
15-19 \\
20-24 \\
25-29 \\
30-34 \\
35-39 \\
40-44 \\
45-49 \\
45-54 \\
50-54 \\
55-59 \\
60-64 \\
65-69 \\
70-74 \\
75-79 \\
80+\end{array}$ & $\begin{array}{l}354 \\
377 \\
347 \\
274 \\
341 \\
395 \\
352 \\
279 \\
173 \\
131 \\
83 \\
66 \\
45 \\
35 \\
25 \\
15\end{array}$ & 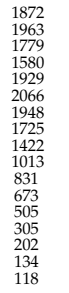 & 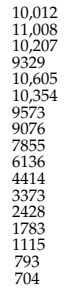 & 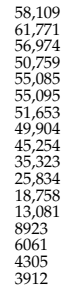 & 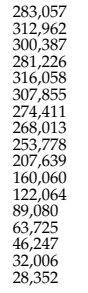 & $\begin{array}{l}4 \\
4 \\
4 \\
4 \\
6 \\
3 \\
2 \\
3 \\
2 \\
2 \\
2 \\
1\end{array}$ & $\begin{array}{l}15 \\
16 \\
17 \\
16 \\
20 \\
10 \\
10 \\
10 \\
9 \\
7 \\
6 \\
5 \\
3 \\
4 \\
2 \\
2 \\
1\end{array}$ & $\begin{array}{l}201 \\
251 \\
229 \\
185 \\
196 \\
149 \\
153 \\
149 \\
130 \\
87 \\
69 \\
60 \\
48 \\
51 \\
34 \\
16 \\
16\end{array}$ & 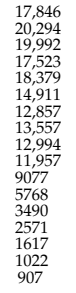 & 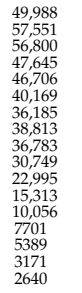 & $\begin{array}{l}47 \\
55 \\
66 \\
50 \\
50 \\
41 \\
37 \\
43 \\
46 \\
34 \\
21 \\
15 \\
18 \\
9 \\
7 \\
5 \\
5\end{array}$ & $\begin{array}{l}567 \\
667 \\
667 \\
549 \\
567 \\
453 \\
453 \\
407 \\
410 \\
329 \\
251 \\
190 \\
162 \\
118 \\
84 \\
47 \\
43\end{array}$ & 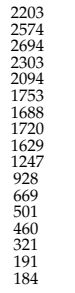 & 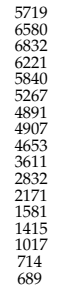 & 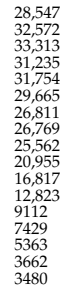 & $\begin{array}{l}65 \\
68 \\
79 \\
89 \\
89 \\
117 \\
67 \\
79 \\
82 \\
70 \\
56 \\
37 \\
28 \\
22 \\
18 \\
17\end{array}$ & $\begin{array}{l}864 \\
873 \\
992 \\
1071 \\
1063 \\
1145 \\
9032 \\
956 \\
955 \\
654 \\
642 \\
465 \\
292 \\
243 \\
218 \\
225\end{array}$ & 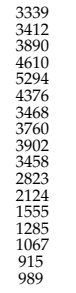 & 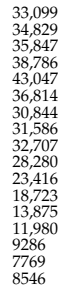 & 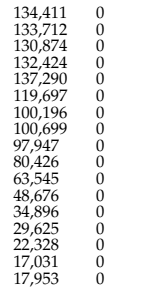 & $\begin{array}{l}0 \\
0 \\
0 \\
0 \\
0 \\
0 \\
0 \\
0 \\
0 \\
0 \\
0 \\
0 \\
0 \\
0 \\
0 \\
0 \\
0 \\
0\end{array}$ & $\begin{array}{ll}0 \\
0 \\
0 \\
0 \\
0 \\
0 \\
0 \\
0 \\
0 \\
0 \\
0 \\
0 \\
0 \\
0 \\
0 \\
0 \\
0 \\
0 \\
0\end{array}$ & $\begin{array}{l}35 \\
41 \\
37 \\
29 \\
18 \\
15 \\
16 \\
13 \\
15 \\
12 \\
12 \\
8 \\
9 \\
6 \\
3 \\
3 \\
3\end{array}$ & $\begin{array}{l}3303 \\
364 \\
341 \\
281 \\
187 \\
146 \\
145 \\
154 \\
110 \\
140 \\
115 \\
87 \\
83 \\
72 \\
64 \\
37 \\
45\end{array}$ & $\begin{array}{l}308 \\
361 \\
337 \\
411 \\
468 \\
470 \\
337 \\
373 \\
332 \\
349 \\
345 \\
280 \\
113 \\
830 \\
89 \\
52\end{array}$ & $\begin{array}{l}911 \\
11108 \\
1134 \\
1178 \\
1422 \\
11400 \\
11992 \\
11022 \\
1055 \\
1068 \\
851 \\
554 \\
404 \\
281 \\
203 \\
1699\end{array}$ & $\begin{array}{l}1210 \\
1493 \\
1531 \\
1717 \\
1918 \\
17999 \\
1489 \\
1399 \\
1433 \\
1392 \\
1914 \\
718 \\
539 \\
371 \\
268 \\
232\end{array}$ & 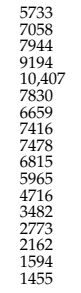 & 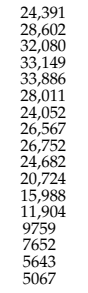 & $\begin{array}{l}20 \\
28 \\
28 \\
25 \\
19 \\
22 \\
23 \\
27 \\
22 \\
11 \\
11 \\
10 \\
7 \\
5 \\
2 \\
2 \\
2\end{array}$ & $\begin{array}{l}53 \\
73 \\
71 \\
69 \\
53 \\
56 \\
57 \\
70 \\
62 \\
38 \\
28 \\
23 \\
25 \\
18 \\
13 \\
4 \\
5\end{array}$ & $\begin{array}{l}93 \\
116 \\
119 \\
112 \\
91 \\
91 \\
86 \\
104 \\
98 \\
68 \\
54 \\
42 \\
38 \\
29 \\
91 \\
9\end{array}$ & 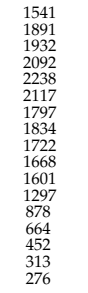 & 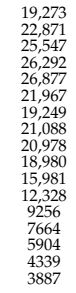 \\
\hline TOTAL & 3606 & 20,065 & 108,768 & 600,803 & $3,346,920$ & 40 & 150 & 2025 & 184,763 & 508,656 & 547 & 5985 & 23,161 & 64,938 & 345,868 & 1059 & 11,875 & 50,267 & 439,434 & $\begin{array}{lll}1,401,729 & 0 \\
\end{array}$ & 0 & 2 & 274 & 2724 & 4963 & 15,110 & 20,044 & 98,680 & 358,909 & 273 & 718 & 1178 & 24,312 & 282,479 \\
\hline
\end{tabular}


Table 4. Number of people living around the seven main asbestos processing/mining sites in Colombia—2018.

\begin{tabular}{|c|c|c|c|c|c|c|c|c|c|c|c|c|c|c|c|c|c|c|c|c|c|c|c|c|c|c|c|c|c|c|c|c|c|c|}
\hline \multirow{2}{*}{$\begin{array}{c}\text { Age } \\
\text { Groups } \\
\text { (Years) }\end{array}$} & \multicolumn{6}{|c|}{ Incolbest-Bogotá } & \multicolumn{4}{|c|}{ Eternit-Sibaté } & \multicolumn{6}{|c|}{ Eternit-Yumbo } & \multicolumn{4}{|c|}{ Eternit-Barranquilla } & \multicolumn{5}{|c|}{ Las Brisas-Campamento } & \multicolumn{5}{|c|}{ Toptec-Manizales } & \multicolumn{4}{|c|}{ Colombit (Etex)-Manizales } \\
\hline & $500 \mathrm{~m}$ & $1000 \mathrm{~m}$ & $2000 \mathrm{~m}$ & $5000 \mathrm{~m}$ & $10,000 \mathrm{~m}$ & $500 \mathrm{~m}$ & $1000 \mathrm{~m}$ & $2000 \mathrm{~m}$ & $5000 \mathrm{~m}$ & $10,000 \mathrm{~m}$ & $500 \mathrm{~m}$ & $1000 \mathrm{~m}$ & $2000 \mathrm{~m}$ & $5000 \mathrm{~m}$ & $10,000 \mathrm{~m}$ & $500 \mathrm{~m}$ & $1000 \mathrm{~m}$ & $2000 \mathrm{~m}$ & $5000 \mathrm{~m}$ & $10,000 \mathrm{~m} 500 \mathrm{~m}$ & $1000 \mathrm{~m}$ & $2000 \mathrm{~m}$ & $5000 \mathrm{~m}$ & $10,000 \mathrm{~m}$ & $500 \mathrm{~m}$ & $1000 \mathrm{~m}$ & $2000 \mathrm{~m}$ & $5000 \mathrm{~m}$ & $10,000 \mathrm{~m}$ & $500 \mathrm{~m}$ & $1000 \mathrm{~m}$ & $2000 \mathrm{~m}$ & $5000 \mathrm{~m}$ & $10,000 \mathrm{~m}$ \\
\hline 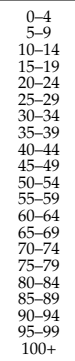 & $\begin{array}{l}364 \\
384 \\
420 \\
5500 \\
612 \\
652 \\
537 \\
498 \\
434 \\
438 \\
3871 \\
2713 \\
113 \\
78 \\
57 \\
30 \\
21 \\
4 \\
2 \\
1\end{array}$ & $\begin{array}{l}1696 \\
1781 \\
1832 \\
2124 \\
2751 \\
27575 \\
2366 \\
2225 \\
1846 \\
1844 \\
1643 \\
1233 \\
9917 \\
159 \\
5515 \\
185 \\
189 \\
27 \\
14 \\
4\end{array}$ & 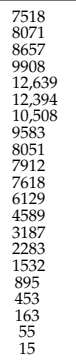 & 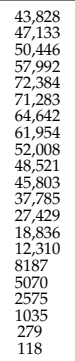 & 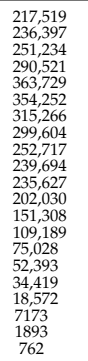 & $\begin{array}{l}1 \\
1 \\
1 \\
1 \\
1 \\
1 \\
1 \\
1 \\
0 \\
1 \\
1 \\
1 \\
0 \\
0 \\
0 \\
0 \\
0 \\
0 \\
0 \\
0 \\
0\end{array}$ & $\begin{array}{l}3 \\
4 \\
3 \\
3 \\
3 \\
3 \\
4 \\
4 \\
4 \\
2 \\
2 \\
2 \\
3 \\
2 \\
1 \\
2 \\
1 \\
0 \\
0 \\
0 \\
0\end{array}$ & 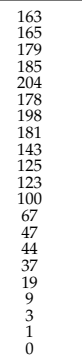 & 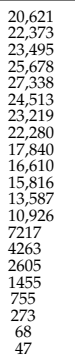 & 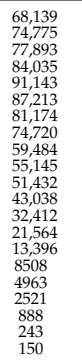 & $\begin{array}{l}30 \\
32 \\
38 \\
49 \\
49 \\
48 \\
41 \\
34 \\
29 \\
29 \\
35 \\
34 \\
24 \\
13 \\
11 \\
12 \\
3 \\
3 \\
1\end{array}$ & $\begin{array}{l}359 \\
384 \\
455 \\
540 \\
558 \\
511 \\
454 \\
444 \\
385 \\
357 \\
355 \\
322 \\
248 \\
173 \\
130 \\
106 \\
60 \\
32 \\
9 \\
4 \\
0\end{array}$ & 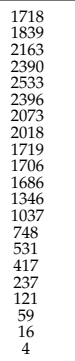 & 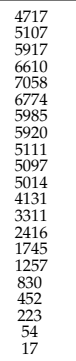 & 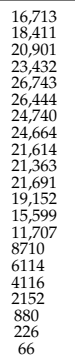 & $\begin{array}{l}62 \\
66 \\
60 \\
74 \\
88 \\
79 \\
66 \\
60 \\
67 \\
60 \\
60 \\
58 \\
47 \\
28 \\
24 \\
17 \\
16 \\
10 \\
4\end{array}$ & $\begin{array}{l}556 \\
611 \\
602 \\
736 \\
812 \\
755 \\
638 \\
666 \\
626 \\
605 \\
618 \\
683 \\
580 \\
430 \\
332 \\
268 \\
214 \\
162 \\
96 \\
30 \\
14 \\
5\end{array}$ & $\begin{array}{l}2097 \\
2181 \\
21210 \\
2806 \\
3166 \\
2550 \\
2561 \\
2608 \\
2313 \\
2190 \\
2430 \\
2363 \\
1997 \\
1960 \\
1904 \\
843 \\
609 \\
376 \\
148 \\
56 \\
20\end{array}$ & 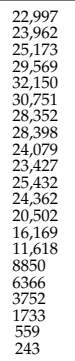 & 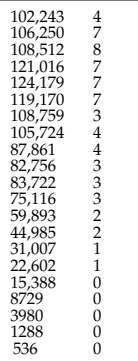 & $\begin{array}{c}19 \\
30 \\
35 \\
32 \\
31 \\
30 \\
15 \\
16 \\
15 \\
14 \\
12 \\
12 \\
9 \\
9\end{array}$ & $\begin{array}{l}43 \\
69 \\
84 \\
75 \\
64 \\
60 \\
34 \\
37 \\
40 \\
34 \\
32 \\
26 \\
19 \\
19 \\
10 \\
7 \\
4 \\
1 \\
0\end{array}$ & $\begin{array}{l}142 \\
215 \\
269 \\
215 \\
175 \\
1753 \\
126 \\
123 \\
190 \\
96 \\
85 \\
68 \\
52 \\
37 \\
22 \\
16 \\
1 \\
1 \\
0 \\
0\end{array}$ & $\begin{array}{l}557 \\
800 \\
949 \\
940 \\
699 \\
601 \\
554 \\
572 \\
453 \\
474 \\
430 \\
4109 \\
309 \\
238 \\
185 \\
143 \\
95 \\
44 \\
15 \\
2 \\
1\end{array}$ & 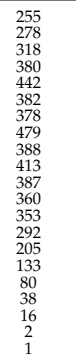 & 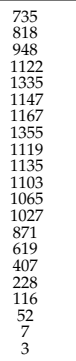 & 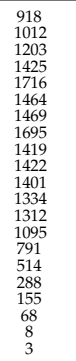 & $\begin{array}{l}4949 \\
5377 \\
6092 \\
7732 \\
9470 \\
8402 \\
7695 \\
8807 \\
7135 \\
7747 \\
7926 \\
7529 \\
6621 \\
5224 \\
3812 \\
2637 \\
1784 \\
1000 \\
4400 \\
89 \\
29\end{array}$ & 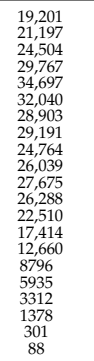 & $\begin{array}{l}10 \\
14 \\
20 \\
19 \\
19 \\
18 \\
15 \\
20 \\
20 \\
16 \\
10 \\
10 \\
6 \\
7 \\
5 \\
4 \\
2 \\
0 \\
0 \\
0 \\
0\end{array}$ & $\begin{array}{l}26 \\
38 \\
48 \\
51 \\
53 \\
44 \\
42 \\
47 \\
49 \\
48 \\
44 \\
29 \\
21 \\
14 \\
17 \\
13 \\
10 \\
5 \\
1 \\
0 \\
0\end{array}$ & $\begin{array}{l}32 \\
48 \\
59 \\
66 \\
66 \\
57 \\
57 \\
57 \\
68 \\
53 \\
57 \\
37 \\
27 \\
18 \\
17 \\
12 \\
6 \\
1 \\
1 \\
0 \\
0\end{array}$ & 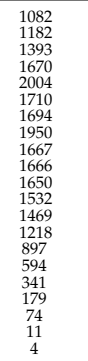 & 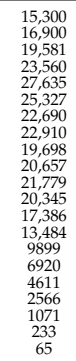 \\
\hline OTAL & 5927 & 26,851 & 122,161 & 729,618 & $3,709,324$ & & 45 & 2172 & 280,981 & 32,838 & 516 & 5886 & 26,758 & 77,746 & 315,439 & 948 & 9409 & 36,527 & 388,444 & 66 & 292 & 658 & 2071 & 8472 & 5582 & 16,378 & 20,715 & 109,599 & 96,661 & 234 & 599 & 746 & 23,986 & 312,615 \\
\hline
\end{tabular}


Table 5. Populations living in the vicinity of two plants in 2005 (Etex and Toptec-Manizales; Incolbest-Bogotá and Eternit-Sibaté).

\begin{tabular}{|c|c|c|c|c|c|c|c|c|c|c|}
\hline \multirow{2}{*}{$\begin{array}{c}\text { Age } \\
\text { Groups } \\
\text { (Years) }\end{array}$} & \multicolumn{5}{|c|}{ ETEX and TOPTEC } & \multicolumn{5}{|c|}{ INCOLBEST and ETERNIT SIBATE } \\
\hline & $500 \mathrm{~m}$ & $1000 \mathrm{~m}$ & $2000 \mathrm{~m}$ & $5000 \mathrm{~m}$ & $10,000 \mathrm{~m}$ & $500 \mathrm{~m}$ & $1000 \mathrm{~m}$ & $2000 \mathrm{~m}$ & $5000 \mathrm{~m}$ & $10,000 \mathrm{~m}$ \\
\hline $0-4$ & $\mathrm{~N} / \mathrm{A}$ & $\mathrm{N} / \mathrm{A}$ & 7 & 1456 & 19,240 & $\mathrm{~N} / \mathrm{A}$ & $\mathrm{N} / \mathrm{A}$ & $\mathrm{N} / \mathrm{A}$ & $\mathrm{N} / \mathrm{A}$ & 3799 \\
\hline $5-9$ & $\mathrm{~N} / \mathrm{A}$ & $\mathrm{N} / \mathrm{A}$ & 6 & 1800 & 22,840 & $\mathrm{~N} / \mathrm{A}$ & $\mathrm{N} / \mathrm{A}$ & $\mathrm{N} / \mathrm{A}$ & $\mathrm{N} / \mathrm{A}$ & 4324 \\
\hline $10-14$ & $\mathrm{~N} / \mathrm{A}$ & $\mathrm{N} / \mathrm{A}$ & 9 & 1839 & 25,513 & $\mathrm{~N} / \mathrm{A}$ & $\mathrm{N} / \mathrm{A}$ & $\mathrm{N} / \mathrm{A}$ & $\mathrm{N} / \mathrm{A}$ & 4026 \\
\hline $15-19$ & $\mathrm{~N} / \mathrm{A}$ & $\mathrm{N} / \mathrm{A}$ & 7 & 2006 & 26,263 & $\mathrm{~N} / \mathrm{A}$ & $\mathrm{N} / \mathrm{A}$ & $\mathrm{N} / \mathrm{A}$ & $\mathrm{N} / \mathrm{A}$ & 3557 \\
\hline $20-24$ & $\mathrm{~N} / \mathrm{A}$ & $\mathrm{N} / \mathrm{A}$ & 8 & 2170 & 26,854 & $\mathrm{~N} / \mathrm{A}$ & $\mathrm{N} / \mathrm{A}$ & $\mathrm{N} / \mathrm{A}$ & $\mathrm{N} / \mathrm{A}$ & 3697 \\
\hline $25-29$ & $\mathrm{~N} / \mathrm{A}$ & $\mathrm{N} / \mathrm{A}$ & 7 & 2050 & 21,941 & $\mathrm{~N} / \mathrm{A}$ & $\mathrm{N} / \mathrm{A}$ & $\mathrm{N} / \mathrm{A}$ & $\mathrm{N} / \mathrm{A}$ & 3436 \\
\hline $30-34$ & $\mathrm{~N} / \mathrm{A}$ & $\mathrm{N} / \mathrm{A}$ & 5 & 1740 & 19,230 & $\mathrm{~N} / \mathrm{A}$ & $\mathrm{N} / \mathrm{A}$ & $\mathrm{N} / \mathrm{A}$ & $\mathrm{N} / \mathrm{A}$ & 3146 \\
\hline $35-39$ & $\mathrm{~N} / \mathrm{A}$ & $\mathrm{N} / \mathrm{A}$ & 5 & 1768 & 21,062 & $\mathrm{~N} / \mathrm{A}$ & $\mathrm{N} / \mathrm{A}$ & $\mathrm{N} / \mathrm{A}$ & $\mathrm{N} / \mathrm{A}$ & 3000 \\
\hline $40-44$ & $\mathrm{~N} / \mathrm{A}$ & $\mathrm{N} / \mathrm{A}$ & 7 & 1661 & 20,957 & $\mathrm{~N} / \mathrm{A}$ & $\mathrm{N} / \mathrm{A}$ & $\mathrm{N} / \mathrm{A}$ & $\mathrm{N} / \mathrm{A}$ & 2576 \\
\hline $45-49$ & $\mathrm{~N} / \mathrm{A}$ & $\mathrm{N} / \mathrm{A}$ & 7 & 1614 & 18,961 & $\mathrm{~N} / \mathrm{A}$ & $\mathrm{N} / \mathrm{A}$ & $\mathrm{N} / \mathrm{A}$ & $\mathrm{N} / \mathrm{A}$ & 1896 \\
\hline $50-54$ & $\mathrm{~N} / \mathrm{A}$ & $\mathrm{N} / \mathrm{A}$ & 6 & 1552 & 15,965 & $\mathrm{~N} / \mathrm{A}$ & $\mathrm{N} / \mathrm{A}$ & $\mathrm{N} / \mathrm{A}$ & $\mathrm{N} / \mathrm{A}$ & 1578 \\
\hline $55-59$ & $\mathrm{~N} / \mathrm{A}$ & $\mathrm{N} / \mathrm{A}$ & 3 & 1261 & 12,318 & $\mathrm{~N} / \mathrm{A}$ & $\mathrm{N} / \mathrm{A}$ & $\mathrm{N} / \mathrm{A}$ & $\mathrm{N} / \mathrm{A}$ & 1266 \\
\hline $60-64$ & $\mathrm{~N} / \mathrm{A}$ & $\mathrm{N} / \mathrm{A}$ & 2 & 849 & 9249 & $\mathrm{~N} / \mathrm{A}$ & $\mathrm{N} / \mathrm{A}$ & $\mathrm{N} / \mathrm{A}$ & $\mathrm{N} / \mathrm{A}$ & 916 \\
\hline $65-69$ & $\mathrm{~N} / \mathrm{A}$ & $\mathrm{N} / \mathrm{A}$ & 2 & 640 & 7659 & $\mathrm{~N} / \mathrm{A}$ & $\mathrm{N} / \mathrm{A}$ & $\mathrm{N} / \mathrm{A}$ & $\mathrm{N} / \mathrm{A}$ & 629 \\
\hline $70-74$ & $\mathrm{~N} / \mathrm{A}$ & $\mathrm{N} / \mathrm{A}$ & 1 & 437 & 5901 & $\mathrm{~N} / \mathrm{A}$ & $\mathrm{N} / \mathrm{A}$ & $\mathrm{N} / \mathrm{A}$ & $\mathrm{N} / \mathrm{A}$ & 408 \\
\hline $75-79$ & $\mathrm{~N} / \mathrm{A}$ & $\mathrm{N} / \mathrm{A}$ & 1 & 303 & 4337 & $\mathrm{~N} / \mathrm{A}$ & $\mathrm{N} / \mathrm{A}$ & $\mathrm{N} / \mathrm{A}$ & $\mathrm{N} / \mathrm{A}$ & 253 \\
\hline $80+$ & $\mathrm{N} / \mathrm{A}$ & $\mathrm{N} / \mathrm{A}$ & 1 & 268 & 3885 & $\mathrm{~N} / \mathrm{A}$ & $\mathrm{N} / \mathrm{A}$ & $\mathrm{N} / \mathrm{A}$ & $\mathrm{N} / \mathrm{A}$ & 223 \\
\hline TOTAL & $\mathrm{N} / \mathrm{A}$ & $\mathrm{N} / \mathrm{A}$ & 82 & 23,416 & 282,175 & $\mathrm{~N} / \mathrm{A}$ & $\mathrm{N} / \mathrm{A}$ & $\mathrm{N} / \mathrm{A}$ & $\mathrm{N} / \mathrm{A}$ & 38,728 \\
\hline
\end{tabular}

Table 6. Populations living in the vicinity of two plants in 2018 (Etex and Toptec-Manizales; Incolbest—Bogotá and Eternit-Sibaté).

\begin{tabular}{|c|c|c|c|c|c|c|c|c|c|c|}
\hline \multirow{2}{*}{$\begin{array}{c}\text { Age } \\
\text { Groups } \\
\text { (Years) }\end{array}$} & \multicolumn{5}{|c|}{ ETEX and TOPTEC } & \multicolumn{5}{|c|}{ INCOLBEST and ETERNIT SIBATE } \\
\hline & $500 \mathrm{~m}$ & $1000 \mathrm{~m}$ & $2000 \mathrm{~m}$ & $5000 \mathrm{~m}$ & $10,000 \mathrm{~m}$ & $500 \mathrm{~m}$ & $1000 \mathrm{~m}$ & $2000 \mathrm{~m}$ & $5000 \mathrm{~m}$ & $10,000 \mathrm{~m}$ \\
\hline $0-4$ & $\mathrm{~N} / \mathrm{A}$ & $\mathrm{N} / \mathrm{A}$ & 2 & 1077 & 15,297 & $\mathrm{~N} / \mathrm{A}$ & $\mathrm{N} / \mathrm{A}$ & $\mathrm{N} / \mathrm{A}$ & $\mathrm{N} / \mathrm{A}$ & 3661 \\
\hline $5-9$ & $\mathrm{~N} / \mathrm{A}$ & $\mathrm{N} / \mathrm{A}$ & 3 & 1176 & 16,895 & $\mathrm{~N} / \mathrm{A}$ & $\mathrm{N} / \mathrm{A}$ & $\mathrm{N} / \mathrm{A}$ & $\mathrm{N} / \mathrm{A}$ & 4070 \\
\hline $10-14$ & $\mathrm{~N} / \mathrm{A}$ & $\mathrm{N} / \mathrm{A}$ & 5 & 1390 & 19,575 & $\mathrm{~N} / \mathrm{A}$ & $\mathrm{N} / \mathrm{A}$ & $\mathrm{N} / \mathrm{A}$ & $\mathrm{N} / \mathrm{A}$ & 4170 \\
\hline $15-19$ & $\mathrm{~N} / \mathrm{A}$ & $\mathrm{N} / \mathrm{A}$ & 6 & 1664 & 23,555 & $\mathrm{~N} / \mathrm{A}$ & $\mathrm{N} / \mathrm{A}$ & $\mathrm{N} / \mathrm{A}$ & $\mathrm{N} / \mathrm{A}$ & 4624 \\
\hline $20-24$ & $\mathrm{~N} / \mathrm{A}$ & $\mathrm{N} / \mathrm{A}$ & 4 & 1999 & 27,627 & $\mathrm{~N} / \mathrm{A}$ & $\mathrm{N} / \mathrm{A}$ & $\mathrm{N} / \mathrm{A}$ & $\mathrm{N} / \mathrm{A}$ & 5256 \\
\hline $25-29$ & $\mathrm{~N} / \mathrm{A}$ & $\mathrm{N} / \mathrm{A}$ & 4 & 1700 & 25,323 & $\mathrm{~N} / \mathrm{A}$ & $\mathrm{N} / \mathrm{A}$ & $\mathrm{N} / \mathrm{A}$ & $\mathrm{N} / \mathrm{A}$ & 5144 \\
\hline $30-34$ & $\mathrm{~N} / \mathrm{A}$ & $\mathrm{N} / \mathrm{A}$ & 3 & 1690 & 22,687 & $\mathrm{~N} / \mathrm{A}$ & $\mathrm{N} / \mathrm{A}$ & $\mathrm{N} / \mathrm{A}$ & $\mathrm{N} / \mathrm{A}$ & 4411 \\
\hline $35-39$ & $\mathrm{~N} / \mathrm{A}$ & $\mathrm{N} / \mathrm{A}$ & 4 & 1944 & 22,908 & $\mathrm{~N} / \mathrm{A}$ & $\mathrm{N} / \mathrm{A}$ & $\mathrm{N} / \mathrm{A}$ & $\mathrm{N} / \mathrm{A}$ & 3926 \\
\hline $40-44$ & $\mathrm{~N} / \mathrm{A}$ & $\mathrm{N} / \mathrm{A}$ & 5 & 1663 & 19,694 & $\mathrm{~N} / \mathrm{A}$ & $\mathrm{N} / \mathrm{A}$ & $\mathrm{N} / \mathrm{A}$ & $\mathrm{N} / \mathrm{A}$ & 3337 \\
\hline $45-49$ & $\mathrm{~N} / \mathrm{A}$ & $\mathrm{N} / \mathrm{A}$ & 3 & 1662 & 20,651 & $\mathrm{~N} / \mathrm{A}$ & $\mathrm{N} / \mathrm{A}$ & $\mathrm{N} / \mathrm{A}$ & $\mathrm{N} / \mathrm{A}$ & 3142 \\
\hline $50-54$ & $\mathrm{~N} / \mathrm{A}$ & $\mathrm{N} / \mathrm{A}$ & 2 & 1647 & 21,775 & $\mathrm{~N} / \mathrm{A}$ & $\mathrm{N} / \mathrm{A}$ & $\mathrm{N} / \mathrm{A}$ & $\mathrm{N} / \mathrm{A}$ & 2840 \\
\hline $55-59$ & $\mathrm{~N} / \mathrm{A}$ & $\mathrm{N} / \mathrm{A}$ & 3 & 1529 & 20,339 & $\mathrm{~N} / \mathrm{A}$ & $\mathrm{N} / \mathrm{A}$ & $\mathrm{N} / \mathrm{A}$ & $\mathrm{N} / \mathrm{A}$ & 2260 \\
\hline $60-64$ & $\mathrm{~N} / \mathrm{A}$ & $\mathrm{N} / \mathrm{A}$ & 2 & 1465 & 17,384 & $\mathrm{~N} / \mathrm{A}$ & $\mathrm{N} / \mathrm{A}$ & $\mathrm{N} / \mathrm{A}$ & $\mathrm{N} / \mathrm{A}$ & 1650 \\
\hline $65-69$ & $\mathrm{~N} / \mathrm{A}$ & $\mathrm{N} / \mathrm{A}$ & 2 & 1217 & 13,483 & $\mathrm{~N} / \mathrm{A}$ & $\mathrm{N} / \mathrm{A}$ & $\mathrm{N} / \mathrm{A}$ & $\mathrm{N} / \mathrm{A}$ & 1258 \\
\hline $70-74$ & $\mathrm{~N} / \mathrm{A}$ & $\mathrm{N} / \mathrm{A}$ & 1 & 896 & 9898 & $\mathrm{~N} / \mathrm{A}$ & $\mathrm{N} / \mathrm{A}$ & $\mathrm{N} / \mathrm{A}$ & $\mathrm{N} / \mathrm{A}$ & 922 \\
\hline $75-79$ & $\mathrm{~N} / \mathrm{A}$ & $\mathrm{N} / \mathrm{A}$ & 1 & 592 & 6920 & $\mathrm{~N} / \mathrm{A}$ & $\mathrm{N} / \mathrm{A}$ & $\mathrm{N} / \mathrm{A}$ & $\mathrm{N} / \mathrm{A}$ & 578 \\
\hline $80-84$ & $\mathrm{~N} / \mathrm{A}$ & $\mathrm{N} / \mathrm{A}$ & 1 & 339 & 4608 & $\mathrm{~N} / \mathrm{A}$ & $\mathrm{N} / \mathrm{A}$ & $\mathrm{N} / \mathrm{A}$ & $\mathrm{N} / \mathrm{A}$ & 323 \\
\hline $85-89$ & $\mathrm{~N} / \mathrm{A}$ & $\mathrm{N} / \mathrm{A}$ & 0 & 179 & 2565 & $\mathrm{~N} / \mathrm{A}$ & $\mathrm{N} / \mathrm{A}$ & $\mathrm{N} / \mathrm{A}$ & $\mathrm{N} / \mathrm{A}$ & 180 \\
\hline $90-94$ & $\mathrm{~N} / \mathrm{A}$ & $\mathrm{N} / \mathrm{A}$ & 0 & 74 & 1071 & $\mathrm{~N} / \mathrm{A}$ & $\mathrm{N} / \mathrm{A}$ & $\mathrm{N} / \mathrm{A}$ & $\mathrm{N} / \mathrm{A}$ & 59 \\
\hline 95-99 & $\mathrm{N} / \mathrm{A}$ & $\mathrm{N} / \mathrm{A}$ & 0 & 11 & 233 & $\mathrm{~N} / \mathrm{A}$ & $\mathrm{N} / \mathrm{A}$ & $\mathrm{N} / \mathrm{A}$ & $\mathrm{N} / \mathrm{A}$ & 17 \\
\hline $100+$ & $\mathrm{N} / \mathrm{A}$ & $\mathrm{N} / \mathrm{A}$ & 0 & 4 & 65 & $\mathrm{~N} / \mathrm{A}$ & $\mathrm{N} / \mathrm{A}$ & $\mathrm{N} / \mathrm{A}$ & $\mathrm{N} / \mathrm{A}$ & 2 \\
\hline TOTAL & $\mathrm{N} / \mathrm{A}$ & $\mathrm{N} / \mathrm{A}$ & 51 & 23,916 & 312,554 & $\mathrm{~N} / \mathrm{A}$ & $\mathrm{N} / \mathrm{A}$ & $\mathrm{N} / \mathrm{A}$ & $\mathrm{N} / \mathrm{A}$ & 51,831 \\
\hline
\end{tabular}




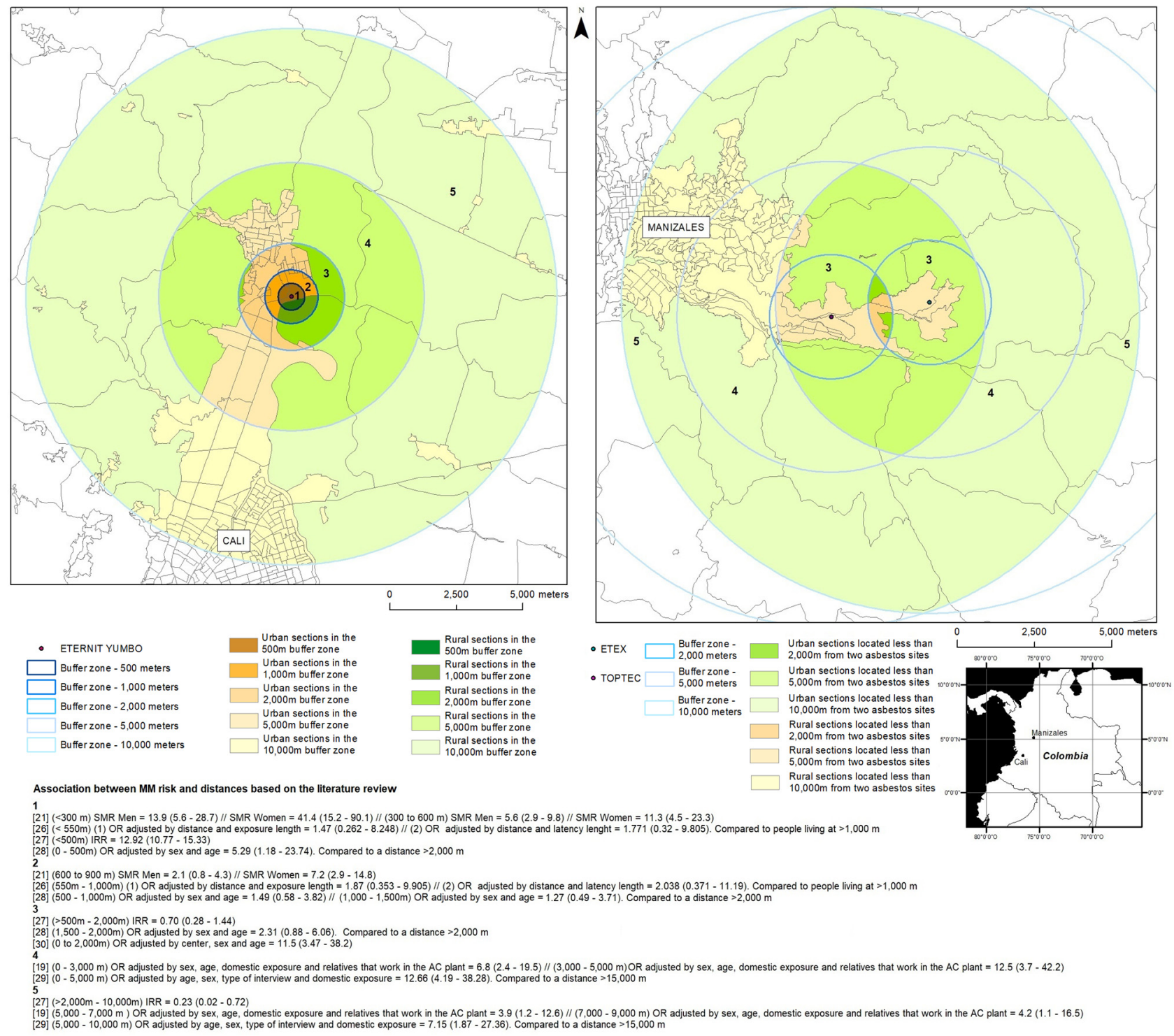

Figure 1. Illustration of the intersection between the buffer zones, based on the relationship between "distance bands and MM risk" extracted from the literature, and the polygons of the MGN (left-Eternit, Yumbo, 2018), highlighting the sectors potentially impacted by atmospheric releases from two sites (right-Etex and Toptec-Manizales, Caldas, 2018).

On the national scale and considering all the sites of interest for this study, the number of people living within a $10 \mathrm{~km}$ radius around these sites is in the millions. Highlighting that no double counting of people was done in Manizales or Bogotá/Sibaté, it is estimated that in 2005, 5,926,382 people lived within $10 \mathrm{~km}$ of the seven sites analyzed, and for 2018 , this number reached $6,724,677$ people. To put the previous figures into context, the population of Colombia was 41,468,384 in 2005 and 48,258,494 in 2018 [24]. Furthermore, the total number of people living within a radius of $1000 \mathrm{~m}$ from all seven sites was 53,904 in 2005, and 59,459 in 2018 (Table 7).

Table 7. Number of people living in the vicinity of the 7 sites analyzed.

\begin{tabular}{cccccc}
\hline Year & $\mathbf{5 0 0} \mathbf{~ m}$ & $\mathbf{1 0 0 0} \mathbf{~ m}$ & $\mathbf{2 0 0 0} \mathbf{~}$ & $\mathbf{5 0 0 0} \mathbf{~}$ & $\mathbf{1 0 , 0 0 0} \mathbf{~ m}$ \\
\hline 2005 & 10,489 & 53,904 & 205,363 & $1,389,788$ & $5,926,382$ \\
2018 & 13,285 & 59,459 & 209,685 & $1,588,528$ & $6,724,677$ \\
\hline
\end{tabular}


It is important to recognize that in the demographic data in the DANE database, there were some polygons with no information, for both 2005 and 2018 (Supplementary Material, Figure S3a,b). It should be noted that the general population census seems to have gained in quality between 2005 and 2018 since the number of polygons without data was drastically reduced. Moreover, it is possible that the lack of data in some polygons has resulted in an underestimation of the populations at risk, based on several official communications with the DANE.

Table 8 shows the number of cases attended to by a healthcare provider at the municipality level between 1 January 2015 and 30 June 2021. A total of 572 registries of health services that attended to mesothelioma cases (i.e., pleural, peritoneal, and pericardial) were identified for the period analyzed in the six municipalities included in the current study (i.e., Campamento, Barranquilla, Bogotá, Manizales, Sibaté, and Yumbo). The age at the moment of the provided healthcare (i.e., by 5-year age groups) was also included in the search (i.e., not shown in Table 8). Because of how the database works, more than one healthcare visit for the same person could be reported for the period consulted. Thus, as is shown in Table 8, for pleural mesothelioma, the total number of people attended to was 350 , but the number of visits was 356. The information presented in Table 8 is focused on mesotheliomas of the pleura (C450), peritoneum (C451), and pericardium (C452), as these are sentinel diseases of asbestos exposure. However, these are not necessarily incident cases, and therefore, risk estimates, such as age-adjusted incident rates, cannot be derived from these figures. Although the cases reported in Table 8 were identified using the municipality of residence of the cases as one of the filters, (that is, instead of the location of the health center where the diagnosis or treatment took place), it is possible, as it was observed in a previous study in Sibate [39], that the residence of the patient is not properly introduced in the SISPRO database. This could explain why in Campamento, a location where an asbestos chrysotile mine operated for more than four decades, no mesothelioma cases were observed, although this could also be explained by the latency and rarity of the disease. Additionally, in the figures consulted, there were mesothelioma cases at unusually young ages (not shown in the table), including cases in the age group of 0-4 years old. Moreover, in the municipality of Yumbo, no pleural, peritoneal, and pericardial mesothelioma cases were reported in women for the time window analyzed, which could be an artifact of the database. Finally, specific information for each case, such as home address and occupation are not reported in SISPRO, which impedes conducting a more sophisticated epidemiological analysis. Thus, the numbers reported in Table 8 should be used with caution.

Table 8. Number of cases of pleural, peritoneal, and pericardial mesotheliomas attended by health providers from 2015 to the first half of 2021 (as of 30 June 2021).

\begin{tabular}{|c|c|c|c|c|c|c|}
\hline \multirow{2}{*}{ Municipality } & \multicolumn{2}{|c|}{ C450-Pleural Mesothelioma } & \multicolumn{2}{|c|}{ C451-Mesothelioma of Peritoneum } & \multicolumn{2}{|c|}{ C452-Mesothelioma of Pericardium } \\
\hline & Females & Males & Females & Males & Females & Males \\
\hline Barranquilla & 38 & 25 & 52 & 15 & 45 & 19 \\
\hline Bogotá & 97 & 163 & 56 & 11 & 4 & 5 \\
\hline Campamento & - & - & - & - & - & - \\
\hline Manizales & 3 & 6 & 6 & 1 & - & 1 \\
\hline Sibaté & 5 & 16 & - & - & - & - \\
\hline Yumbo & - & 3 & - & 1 & - & - \\
\hline Total & 143 & 213 & 114 & 28 & 49 & 25 \\
\hline
\end{tabular}

Source: SISPRO-accessed on 5 October 2021.

\section{Discussion}

The operation of seven facilities that process asbestos in Colombia, the first one beginning in 1942, has left a negative legacy of asbestos-containing products in the country, as well as people occupationally and environmentally exposed to asbestos. Furthermore, a malignant pleural mesothelioma cluster involving, so far, mostly members of the general 
population was found in Sibaté, where the first asbestos cement facility of the country was built [39]. The potential presence of an excess number of asbestos-related diseases in other regions in Colombia where asbestos processing facilities have operated has not yet been assessed. In the current study, the number of people living in the vicinity of asbestos processing plants or the mine ranges from approximately 10,000 inhabitants within the $500 \mathrm{~m}$ radii to about 6 million people within the $10 \mathrm{~km}$ radii, the latter representing about $14 \%$ of the country's total population. This clearly indicates the importance of establishing surveillance programs for both asbestos-related diseases and asbestos exposure sources in regions where asbestos processing plants and the mine have operated, something that has become more relevant considering the large number of people living nearby these facilities.

This analysis, as rigorous as it was, should be read only as an estimation exercise, considering that it was based on distances at which an increased risk of ARD has been observed in other locations of the world, but no asbestos exposure campaigns have been conducted in Colombia in the surroundings of asbestos facilities or the mine, similarly to what happens in most low- and middle-income countries. Only sampling campaigns could prove if the real exposure to asbestos fibers in these areas occurred, as it has been exemplified by several cases, including serpentine quarries in Italy [40], an asbestos textile factory in Indonesia [41], and an asbestos cement sheet manufacturing plant in India [42]. However, since asbestos has already been banned in Colombia, the opportunity to measure asbestos concentrations in the air surrounding asbestos processing facilities and the mine is no longer available. This shows the importance of measuring asbestos fiber concentrations, implementing air quality models, and implementing asbestos-related disease surveillance programs in regions of the world where asbestos processing facilities or asbestos mines are still operating.

Another point to mention is that the population residing around the Campamento mine has increased much more in proportion than those around the other sites during the 2005-2018 period (Tables 3 and 4), indicating that the 2005 census may have missed inhabitants of the rural area (Supplementary Material, Figure S3a). This is important because there was a significant increase in asbestos production from the mine between the years 2005 and 2018. In 2005 and the five years preceding the 2005 census, the asbestos production of the mine ranged between 4246 and 6539 tons of asbestos per year [43,44]. In 2018 and the five years preceding the 2018 census, the production of the mine increased to a range between 3550 and 13,239 tons of asbestos per year, an increase that coincides with a new mine owner taking over in 2013 [45]. Thus, what seems to be a significant increase in the population at risk in the region of the Campamento mine is consistent with a significant increase in chrysotile fiber production.

The present study has the following limitations. First, in some cases, the polygons of the MGN were not entirely included under the radii of the different buffers. When the buffer radius crossed a polygon, a coefficient was applied to the initial population size of the complete polygon, a coefficient that was estimated based on the area of the polygon covered by the buffer radius, as explained in Section 2. This approach assumes that the distribution of the population is homogeneous across a polygon, which could lead to small errors if this is not the case. However, this small error is certainly limited for two reasons. First, the most populated urban areas also correspond to the urban sections that tend to have a more homogeneous distribution of the population. Moreover, the MGN urban polygons have small areas, and therefore, as the buffer radius increases, more MGN polygons will be completely included within the buffers-reducing the need to apply the correction. For example, within a radius of $500 \mathrm{~m}$ around the Eternit plant in Barranquilla in 2005, 0 MGN polygons of urban sections out of 7 are entirely within the buffer (i.e., the correction had to be applied to seven polygons-100\%), 1 out of 12 are entirely within the $1000 \mathrm{~m}$ radius buffer, 21 out of 49 are entirely within the $2000 \mathrm{~m}$ radius buffer, 209 out of 259 are entirely within the $5000 \mathrm{~m}$ radius buffer, and 711 out of 750 are entirely within the $10,000 \mathrm{~m}$ radius buffer (i.e., the correction was applied to 39 polygons- $5.2 \%$ ). Second, 
rural sections or sectors-although more extensive-are also much less populated than the urban sections or sectors; the error will, therefore, be limited in this case as well.

Another limitation is a possible underestimation of the population at risk as a result of certain sectors without demographic data (Supplementary Material, Figure S3a,b). Some scenarios-especially in 2005-have as much as 50\% of the polygons without data in rural areas (i.e., the mine in Campamento with one polygon out of two). For the same year, in urban areas, the largest percentage of the polygons with no data was $21.4 \%$, in the $1000 \mathrm{~m}$ band around Eternit, Yumbo. Several official communications with the DANE have helped explain this phenomenon, which was the result of personal safety concerns of census officials in these areas that prevented them from using, in some cases, geolocation equipment to record census information. In addition, it was identified that some urban sections - in urban areas, as confirmed by satellite images-sometimes appeared to not have resident populations. The DANE indicates, from an example we provided, that some areas correspond to areas with buildings that are not housing units (e.g., industrial zones), which could explain why sometimes these areas do not have residents. This is notably the case near Eternit, Yumbo. Nevertheless, this lack of data tended to decrease between the censuses, decreasing from $4.6 \%$ of polygons without data in the $10,000 \mathrm{~m}$ band for all the sites studied in 2005 to $1.4 \%$ in 2018 (Supplementary Material, Figure S3a,b). Finally, it is possible to note a relative drop in the population in the 500,1000, and 10,000 $\mathrm{m}$ radii around Eternit, Yumbo between 2005 and 2018, which the DANE explains resulted from the degree of geographical precision.

Another aspect to mention is the narrow temporal window of the analysis conducted (years 2005 to 2018). Indeed, since the asbestos industry began operations in Colombia in 1942, it would have been interesting to replicate this work with previous censuses. The DANE confirmed that only these two censuses are available online. Furthermore, for previous censuses, the microdata from the 1993 General Population Census is not easily available and can only be consulted in the specialized external processing room of the DANE. Thus, in future studies, it would be important to determine the feasibility of including the 1993 census in the analysis.

Another limitation of this method was the use of concentric areas in the analysis. Although it was based on distances reported in studies conducted in other regions of the world, the perfectly concentric character of the potential dispersion of asbestos fibers is geometrically convenient. The topographical context and local meteorology play a role in the dispersion of asbestos fibers, and as a result, in the risk of asbestos exposure. The importance of prevailing winds in the simulation of asbestos dispersion from factories producing asbestos cement products [21,46] or asbestos textiles [41] has been clearly established and indicates that such dispersion is not necessarily concentric.

Finally, this work-recognizing the significance that the potential dispersion of asbestos from industrial sources may play in its exposure to the population-must not obscure other exposure scenarios. As has been reported by other authors in the past, a recent study reaffirms the possibility of having other sources of asbestos exposure in the area surrounding an asbestos facility, including both domestic and occupational settings [29].

In the case of Sibate (Colombia), where an asbestos facility operated for more than seven decades, massive disposal of waste contaminated with friable asbestos in the urban area of the municipality was identified $[39,47,48]$, which is something that may have occurred around other asbestos processing facilities. This point is particularly critical in a country like Colombia, where waste regulations are fairly recent [49-51] and the degree of compliance and enforcement by the authorities is unknown. In this context, it is important to implement health surveillance programs for asbestos workers and their families, determine if there are still active sources that could be releasing asbestos fibers into the environment, and study the potential presence of contaminated sites because of the improper disposal of residues contaminated with asbestos, especially for the areas where the asbestos facilities and the mine operated in Colombia. Because of the lack of 
enforcement and surveillance of asbestos processing facilities in low- and middle-income countries, the vast majority of studies have been conducted in high-income countries.

\section{Conclusions}

In this study, we estimated the number of people living in Colombia in close proximity to asbestos processing facilities and the only asbestos mine in the country (up to a distance of $10 \mathrm{~km}$ ). Within these distances, studies conducted in other parts of the world have found an increased risk of ARD, especially mesothelioma. However, these risk estimates cannot be directly applied to the Colombian population because local aspects, such as meteorological conditions, particular attributes of each plant (i.e., type of products manufactured, volumes and asbestos types used, production process, and emission control strategies in place), and local topography, among others, can modify the risk experienced by the population living around each plant. The results of the current study reaffirm the importance of implementing mesothelioma registries and other surveillance strategies for asbestos-related diseases in Colombia, especially in the regions where asbestos processing plants or the mine are located, and the urgent need to develop and implement a National Plan for the Elimination of Asbestos-related Diseases.

Supplementary Materials: The following are available online at https:/ /www.mdpi.com/article/ 10.3390/ijerph182413297/s1, Figure S1: Protocol to access and process census data from Colombia, Figure S2: Overview of the GIS procedure, and Figure S3a: Number of polygons without data in 2005 for each distance scenario, and Figure S3b: Number of polygons without data in 2018 for each distance scenario.

Author Contributions: Conceptualization, B.L., M.F.C.-G., M.G., J.M.L., J.C.G.-B. and L.B.-A.; Data curation, B.L.; Formal analysis, B.L., M.F.C.-G., M.G., J.M.L., L.M.S.-C. and J.P.R.-B.; Funding acquisition, B.L., M.F.C.-G., J.M.L., J.C.G.-B., E.C.R. and J.P.R.-B.; Investigation, B.L.; Methodology, B.L., M.F.C.-G., J.M.L., L.M.S.-C., L.B.-A. and J.P.R.-B.; Project administration, B.L. and J.P.R.-B.; Supervision, J.P.R.-B.; Writing—original draft, B.L.; Writing—review \& editing, M.F.C.-G., M.G., J.M.L., L.M.S.-C., J.C.G.-B., L.B.-A., E.C.R. and J.P.R.-B. All authors have read and agreed to the published version of the manuscript.

Funding: This study was funded with the grant "Research for the Achievement of Sustainable Development Goals, 2019", financed by three Colombian universities: Universidad de Los Andes, Pontificia Universidad Javeriana, and Universidad del Rosario. The French National Research Institute for Sustainable Development (IRD) also supported the study.

Data Availability Statement: Data bases used can be found at: 1-DANE. Censos Nacionales de Población y Vivienda. 2020. Available online: http://systema59.dane.gov.co/bincol/rpwebengine. exe/PortalAction?lang=esp (accessed on 3 November 2021). 2-DANE. Marco Geoestadistico Nacional-MGN. 2020. Available online: https://geoportal.dane.gov.co/servicios/descarga-ymetadatos/descarga-mgn-marco-geoestadistico-nacional/ (accessed on 3 November 2021).

Acknowledgments: The authors thank the DANE teams for their availability to answer our numerous questions. All authors acknowledge financial provided by the Vice Presidency for Research \& Creation publication fund at the Universidad de los Andes.

Conflicts of Interest: Benjamin Lysaniuk, María Fernanda Cely-García, Margarita Giraldo, Joan M. Larrahondo, Laura Marcela Serrano-Calderón, Juan Carlos Guerrero-Bernal, Leonardo BricenoAyala, Esteban Cruz Rodriguez, and Juan Pablo Ramos-Bonilla have no financial conflict of interest to declare. Juan Pablo Ramos-Bonilla provided expert opinion in the Colombian Senate in the discussion of a law banning asbestos in the country. 


\section{References}

1. ATSDR. Toxicological Profile for Asbestos. U.S. Department of Health and Human Services. Atlanta; 2001. Available online: http:/ / www.atsdr.cdc.gov/toxprofiles/tp61.pdf (accessed on 3 November 2021).

2. CDC. Asbestos Fibers and Other Elongate Mineral Particles: State of the Science and Roadmap for Research. Revised Edition. Curr. Intell. Bull. 2011, 62. Available online: https://www.cdc.gov/niosh/docs/2011-159/pdfs/2011-159.pdf (accessed on 3 November 2021).

3. Campbell, W.J.; Blake, R.L.; Brown, L.L.; Cather, E.E.; Sjoberg, J.J. Selected Silicate Minerals and Their Asbestiform Varieties: Mineralogical Definitions and Identification-Characterization; Dept. of the Interior, Bureau of Mines: Washington, WA, USA, 1977.

4. Virta, R.L. Asbestos: Geology, Mineralogy, Mining, and Uses by Open-File Report 02-149; US Department of the Interior, US Geological Survey: Washington, DC, USA, 2002; pp. 1-28. Available online: http://pubs.usgs.gov/of/2002/of02-149/of02-149.pdf (accessed on 3 November 2021).

5. $\quad$ Ross, M.; Langer, A.M.; Nord, G.L.; Nolan, R.P.; Lee, R.J.; Van Orden, D.; Addison, J. The mineral nature of asbestos. Regul. Toxicol. Pharmacol. 2008, 52 (Suppl. 1), S26-S30. [CrossRef]

6. Addison, J.; McConnell, E.E. A review of carcinogenicity studies of asbestos and non-asbestos tremolite and other amphiboles. Regul. Toxicol. Pharmacol. 2008, 52, S187-S199. [CrossRef] [PubMed]

7. IARC. IARC Monographs on the Evaluation of Carcinogenic Risks to Humans. Asbestos (Chrysotile, Amosite, Crocidolite, Tremolite, Actinolite, and Anthophyllite). IARC Monogr.—Vol. 100C. 2012, pp. 219-294. Available online: http://monographs. iarc.fr/ENG/Monographs/vol100C/index.php (accessed on 3 November 2021).

8. Kang, D.M.; Kim, J.E.; Kim, Y.K.; Lee, H.H.; Kim, S.Y. Occupational burden of Asbestos-related diseases in Korea, 1998-2013: Asbestosis, mesothelioma, lung Cancer, laryngeal Cancer, and ovarian Cancer. J. Korean Med. Sci. 2018, 33, e226. [CrossRef] [PubMed]

9. Pasetto, R.; Terracini, B.; Marsili, D.; Comba, P. Occupational burden of asbestos-related cancer in Argentina, Brazil, Colombia and Mexico. Ann. Glob. Health 2014, 80, 263-268. [CrossRef] [PubMed]

10. Ferrante, D.; Mirabelli, D.; Tunesi, S.; Terracini, B.; Magnani, C. Pleural mesothelioma and occupational and non-occupational asbestos exposure: A case-control study with quantitative risk assessment. Occup. Environ. Med. 2016, 73, 147-153. [CrossRef]

11. Carlin, D.J.; Larson, T.C.; Pfau, J.C.; Gavett, S.H.; Shukla, A.; Miller, A.; Hines, R. Current research and opportunities to address environmental asbestos exposures. Environ. Health Perspect 2015, 123, A194-A197. [CrossRef]

12. Marsili, D.; Terracini, B.; Santana, V.S.; Ramos-Bonilla, J.P.; Pasetto, R.; Mazzeo, A.; Loomis, D.; Comba, P.; Algranti, E. Prevention of asbestos related disease in countries currently using asbestos. Int. J. Environ. Res. Public Health 2016, 13, 494. [CrossRef] [PubMed]

13. Moon, E.K.; Son, M.; Jin, Y.W.; Park, S.; Lee, W.J. Variations of lung cancer risk from asbestos exposure: Impact on estimation of population attributable fraction. Ind. Health 2013, 51, 128-133. [CrossRef]

14. Goldberg, M.; Luce, D. The health impact of nonoccupational exposure to asbestos: What do we know? Eur. J. Cancer Prev. 2009, 18, 489-503. [CrossRef]

15. IBAS. Current Asbestos Bans. 2021. Available online: http://ibasecretariat.org/alpha_ban_list.php (accessed on 20 January 2021)

16. Congreso. Ley 1968 Por el cual se Prohíbe el uso de Asbesto en el Territorio Nacional y se Establecen Garantías de Proteccón a la Salud de los Colombianos. 2019. Available online: https:// dapre.presidencia.gov.co/normativa/normativa/LEY\%201968\%2 0DEL\%2011\%20DE\%20JULIO\%20DE\%202019.pdf (accessed on 15 April 2021).

17. ILO-WHO. Outline for the Development of National Programmes for Elimination of Asbestos Related Diseases; ILO-WHO: Geneva, Switzerland, 2007.

18. Magnani, C.; Terracini, B.; Ivaldi, C.; Botta, M.; Mancini, A.; Andrion, A. Pleural malignant mesothelioma and non-occupational exposure to asbestos in Casale Monferrato, Italy. Occup. Environ. Med. 1995, 52, 362-367. [CrossRef]

19. Maule, M.M.; Magnani, C.; Dalmasso, P.; Mirabelli, D.; Merletti, F.; Biggeri, A. Modeling mesothelioma risk associated with environmental asbestos exposure. Environ. Health Perspect. 2007, 115, 1066-1071. [CrossRef] [PubMed]

20. Kumagai, S.; Kurumatani, N. Asbestos fiber concentration in the area surrounding a former asbestos cement plant and excess mesothelioma deaths in residents. Am. J. Ind. Med. 2009, 52, 790-798. [CrossRef]

21. Kurumatani, N.; Kumagai, S. Mapping the risk of mesothelioma due to neighborhood asbestos exposure. Am. J. Respir. Crit. Care Med. 2008, 178, 624-629. [CrossRef]

22. Musk, A.; Reid, A.; Olsen, N.; Hobbs, M.; Armstrong, B.; Franklin, P.; Hui, J.; Layman, L.; Merler, E.; Brims, F.; et al. The Wittenoom legacy. Int. J. Epidemiol. 2020, 49, 467-476. [CrossRef]

23. Naik, S.; Lewin, M.; Young, R.; Dearwent, S.; Lee, R. Mortality from asbestos-associated disease in Libby, Montana 1979-2011. J. Expo. Sci. Environ. Epidemiol. 2017, 27, 207-213. [CrossRef]

24. DANE. Censos Nacionales de Población y Vivienda. 2020. Available online: http://systema59.dane.gov.co/bincol/rpwebengine. exe/PortalAction?lang=esp (accessed on 15 May 2021).

25. DANE. Marco Geoestadistico Nacional-MGN. 2020. Available online: https://geoportal.dane.gov.co/servicios/descarga-ymetadatos / descarga-mgn-marco-geoestadistico-nacional/ (accessed on 15 May 2021).

26. Vimercati, L.; Cavone, D.; Delfino, M.; Caputi, A.; De Maria, L.; Sponselli, S.; Corrado, V.; Ferri, G.; Serio, G. Asbestos Air Pollution: Description of a Mesothelioma Cluster Due to Residential Exposure from an Asbestos Cement Factory. Int. J. Environ. Res. Public Health 2020, 17, 2636. [CrossRef] 
27. Tarrés, J.; Albertí, C.; Martínez-Artés, X.; Abós-Herràndiz, R.; Rosell-Murphy, M.; García-Allas, I.; Krier, I.; Cantarell, G.; Gallego, M.; Canela-Soler, J.; et al. Pleural mesothelioma in relation to meteorological conditions and residential distance from an industrial source of asbestos. Occup. Environ. Med. 2013, 70, 588-590. [CrossRef]

28. Musti, M.; Pollice, A.; Cavone, D.; Dragonieri, S.; Bilancia, M. The relationship between malignant mesothelioma and an asbestos cement plant environmental risk: A spatial case-control study in the city of Bari (Itali). Int. Arch. Occup. Environ. Health 2009, 82, 489-497. [CrossRef] [PubMed]

29. Airoldi, C.; Magnani, C.; Lazzarato, F.; Mirabelli, D.; Tunesi, S.; Ferrante, D. Environmental asbestos exposure and clustering of malignant mesothelioma in community: A spatial analysis in a population-based case-control study. Environ. Health 2021, 20, 1-13. [CrossRef] [PubMed]

30. Magnani, C.; Agudo, A.; González, C.A.; Andrion, A.; Calleja, A.; Chellini, E.; Dalmasso, P.; Escolar, A.; Hernández, S.; Ivaldi, C.; et al. Multicentric study on malignant pleural mesothelioma and non-occupational exposure to asbestos. Br. J. Cancer 2000, 83, 104-111. [CrossRef] [PubMed]

31. Price, B. Exposure to airborne amphibole structures and health risks: Libby, Montana. Regul. Toxicol. Pharmacol. 2008, 52, S97-S109. [CrossRef]

32. Noonan, C.; Conway, K.; Landguth, E.; McNew, T.; Linker, L.; Pfau, J.; Black, B.; Szeinuk, J.; Flores, R. Multiple pathway asbestos exposure assessment for a Superfund community. J. Expo. Sci. Environ. Epidemiol. 2015, 25, 18-25. [CrossRef] [PubMed]

33. Rogers, A.; Nevill, M. Occupational and environmental mesotheliomas due to crocidolite mining activities in Wittenoom, Western Australia. Scand. J. Work Environ. Health 1995, 21, 259-264. [CrossRef]

34. Hansen, J.; De Klerk, N.H.; Musk, A.W.; Hobbs, M.S. Environmental exposure to crocidolite and mesothelioma: Exposure-response relationship. Am. J. Respir. Crit. Care Med. 1998, 157, 69-75. [CrossRef] [PubMed]

35. Kielkowski, D.; Nelson, G.; Rees, D. Risk of mesothelioma from exposure to crocidolite asbestos: A 1995 update of a South African mortality study. Occup. Environ. Med. 2000, 57, 563-567. [CrossRef]

36. Braun, L.; Kisting, S. Asbestos-related disease in South Africa: The social production of an invisible epidemic. Am. J. Public Health 2006, 96, 1386-1396. [CrossRef] [PubMed]

37. Noonan, C. Environmental asbestos exposure and risk of mesothelioma. Ann. Transl. Med. 2017, 5, 234. [CrossRef]

38. Datosmacro.com Colombia-Piramide de Población. 2020. Available online: https://datosmacro.expansion.com/demografia/ estructura-poblacion/colombia (accessed on 15 February 2021).

39. Ramos-Bonilla, J.P.; Cely-García, M.F.; Giraldo, M.; Comba, P.; Terracini, B.; Pasetto, R.; Marsili, D.; Ascoli, V.; Lysaniuk, B.; Rodríguez, M.C.; et al. An asbestos contaminated town in the vicinity of an asbestos-cement facility: The case study of Sibaté, Colombia. Environ. Res. 2019, 176, 108464. [CrossRef] [PubMed]

40. Cattaneo, A.; Somigliana, A.; Gemmi, M.; Bernabeo, F.; Savoca, D.; Cavallo, D.; Bertazzi, P. Airborne concentrations of chrysotile asbestos in serpentine quarries and stone processing facilities in Valmalenco. Italy. Ann. Occup. Hyg. 2012, 56, 671-683. [CrossRef]

41. Kang, D.; Hwang, Y.; Choi, Y.; Kim, S.; Kim, Y. Monitoring and Simulating Environmental Asbestos Dispersion from a Textile Factory. Int. J. Environ. Res. Public Health 2018, 15, 1398. [CrossRef] [PubMed]

42. Ansari, F.; Bihari, V.; Rastogi, S.; Ashquin, M.; Ahmad, I. Environmental health survey in asbestos cement sheets manufacturing industry. Indian J. Occup. Environ. Med. 2007, 11, 15-20. [CrossRef] [PubMed]

43. Written answer to request from Agencia Nacional de Minería Resumen Histórico de la Producción de "Fibra de Asbesto". Respuesta No 20173210263811 de la ANM al Derecho de Petición 20175510216022. 2017.

44. Written answer to request from Agencia Nacional de Minería Producción asbesto en el municipio de Campamento en el 2018. Respuesta No 20203210306581 de la ANM al Derecho de Petición 20201000569462. 2020.

45. Semana La Polémica Mina Que Revivió en Antioquia. Recuperado el 3 de Agosto de 2020; Publicaciones Semana S.A.: Bogotá, Colombia, 7 October 2013.

46. Counil, E.; Daniau, C.; Isnard, H. Étude de Santé Publique Autour D'une Ancienne Usine de Broyage D'amiante: Le Comptoir des Minéraux et Matières Premières à Aulnay-Sous-Bois (Seine-Saint-Denis)—Pollution Environnementale Entre 1938 et 1975: Impacts Sanitaires et Recommandations. 2007. Available online: https://www.santepubliquefrance.fr/regions/ile-defrance/documents / rapport-synthese/2007 / etude-de-sante-publique-autour-d-une-ancienne-usine-de-broyage-d-amiante-lecomptoir-des-mineraux-et-matieres-premieres-a-aulnay-sous-bois-seine2 (accessed on 15 April 2021).

47. Cely-García, M.F.; Lysaniuk, B.; Pasetto, R.; Ramos-Bonilla, J.P. The challenges of applying an Activity-Based Sampling methodology to estimate the cancer risk associated with asbestos contaminated landfilled zones. Environ. Res. 2020, 181, 1-10. [CrossRef] [PubMed]

48. Lysaniuk, B.; Cely-García, M.; Mazzeo, A.; Marsili, D.; Pasetto, R.; Comba, P.; Ramos-Bonilla, J.P. Where are the landfilled zones? Use of historical geographic information and local spatial knowledge to determine the location of underground asbestos contamination in Sibaté (Colombia). Environ. Res. 2020, 191, 1-14. [CrossRef]

49. Ministerio de Ambiente, Vivienda y Desarrollo Territorial. Decreto Número 4741 por el Cual se Reglamenta Parcialmente la Prevención y Manejo de los Residuos o Desechos Peligrosos Generados en el Marco de la Gestión Integral. 2005. Available online: http:/ / www.ideam.gov.co/documents/51310/526371/Decreto+4741+2005+PREVENCION+Y+MANEJO+ DE+REIDUOS+PELIGROSOS+GENERADOS+EN+GESTION+INTEGRAL.pdf/491df435-061e-4d27-b40f-c8b3afe25705 (accessed on 15 June 2021). 
50. Ministerio de Ambiente, Vivienda y Desarrollo Territorial. Resolución Número 1362 por la Cual se Establece los Requisitos y el Procedimiento para el Registro de Generadores de Residuos o Desechos Peligrosos, a que Hacen Referencia los Artículos $27^{\circ}$ y $28^{\circ}$ del Decreto 4741 del 30 de Diciembre de 2005. Available online: http:/ / www.ideam.gov.co/documents/51310/526371 /Resolucion+1362+2007++REQUISITOS+Y+PROCEDIMIENTOS+PARA+REG+DE+GENERADORES+DE+RESPEL.pdf/cdd6 d851-013b-4bea-adf6-addec449f32b (accessed on 15 June 2021).

51. Ministerio de Salud y Protección Social. Resolución Número 007 por la Cual se Adopta el Reglamento de Higiene y Seguridad del Crisotilo y Otras Fibras de Uso Similar. 2011. Available online: https://www.minsalud.gov.co/sites/rid/Lists/BibliotecaDigital/ RIDE/DE/DIJ/resolucion-007-2011.pdf (accessed on 15 June 2021). 